\section{Photoactuated printing}

Adv. Mater. http://doi.org/gcqsvn (2017)

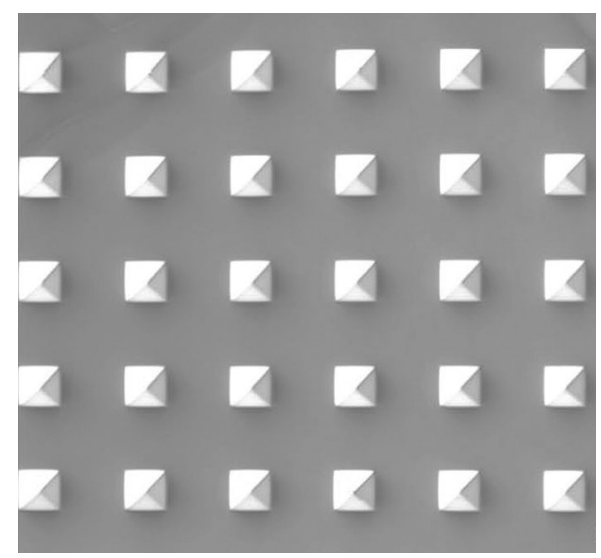

Credit: Wiley

By using a highly uniform distribution of functionalized carbon nanotubes (CNTs) in a polydimethylsiloxane (PDMS) matrix, Zhongjie Huang and co-workers have made a two-dimensional array of photoactuated pens with an actuation efficiency of $200 \mathrm{~nm} \mathrm{~mW}^{-1}$. Their pen arrays were fabricated by curing the moulded composite paste on a silicon wafer that contained an array of microfabricated pyramidal holes. The pitch and base length of the pyramidal holes are $60 \mu \mathrm{m}$ and $20 \mu \mathrm{m}$, respectively. A pyramidal pen can locally expand on light irradiation with a focal spot of $2 \mathrm{~mm}$ in radius and an average light intensity of $100 \mathrm{~mW} \mathrm{~cm}^{-2}$. On irradiation, the local deformation moves the pen out of plane into contact with a substrate so that local material transfer occurs. This actuation occurs locally, meaning pens outside the illumination area do not print. The selected pen can move more than $3 \mu \mathrm{m}$ out of plane, enabling controllable and high-quality printing.

https://doi.org/10.1038/s41566-018-0126-3

\section{QUANTUM PHYSICS}

\section{Breaking symmetry}

Phys. Rev. A 97, 013802 (2018)

Ningyuan Jia and colleagues from the University of Chicago, USA, have developed a device to break time-reversal symmetry using a four-mirror running-wave resonator. They twist the resonator out of plane by $6^{\circ}$ to break inversion symmetry. An optically active ensemble with ${ }^{87} \mathrm{Rb}$ atoms is placed in the resonator to provide a resonatorenhanced atomic Faraday effect that breaks the time-reversal symmetry. These two broken symmetries result in a frequency shift of $9.4 \mathrm{MHz}$ between the forward- and backward-propagating modes. Choosing the incident beam's polarization isolates one of the propagating modes. The team obtained an isolation of $20 \mathrm{~dB}$ for the backward mode, with $83 \%$ relative forward cavity transmission. Cavity Rydberg electromagnetically induced transparency (EIT) spectra were measured for forward- $\left(\sigma^{+}\right)$and backward-propagating polarized $\left(\sigma^{-}\right)$light. When the cavity was in resonance with the $\sigma^{+}$light, probing in the forward direction revealed a standard EIT spectrum, while probing in the backward direction revealed only a weak Fano feature

\title{
PLASMONICS
}

\section{Versatile plasmomechanical systems Optica 5, 71-79(2018)}

Brian Roxworthy and Vladimir Aksyuk from the National Institute of Standards and Technology, USA, have now demonstrated a plasmomechanical system that can localize optothermomechanical interactions to subdiffraction volumes. Their approach is based on electrically tunable localized-gap plasmon resonators (LGPRs) that use electrostatic actuation. A point-like LGPR is embedded into a fully functional nanoelectromechanical system that is a simple silicon nitride cantilever design. The cantilever is approximately $165 \mathrm{~nm}$ thick and has on top an approximately $15-\mathrm{nm}$-thick gold layer. The cantilever is suspended above, with a nominal gap of $20 \mathrm{~nm}$, an underlying stationary gold pad and can be electrostatically actuated by applying a voltage between the top gold layer and the pad. The LGPR is defined by a 40-nm-thick gold cuboid embedded in the cantilever's underside, the gold pad and the gap, and it mediates both optomechanical and thermomechanical coupling via its spatial location. The team shows that the thermomechanical coupling can induce self-oscillation of the nanomechanical device and can be injection-locked to a small-amplitude mechanical stimulus. Changing the plasmonic gap tunes the coupling and controls the threshold power of the oscillator. The findings will be of use for facile electrooptic modulation, nanomechanical sensing and reconfigurable metasurfaces.

for cavity tuning. These findings may be useful for creating new photonics materials and switchable narrow-band optical isolators. $\mathrm{NH}$

https://doi.org/10.1038/s41566-018-0127-2

\section{TERAHERTZ PHOTONICS}

\section{Organic opportunities \\ ACS Photon. http://doi.org/ckb7 (2018)}

A new breed of organic crystal is showing great promise for the efficient generation and detection of terahertz waves. Matteo Savoini and co-workers have discovered that diphenylfluorenone (DPFO) crystals have an extremely large electro-optical coefficient of $74 \mathrm{pm} \mathrm{V}^{-1}$ at around $1.5 \mathrm{THz}$. In comparison, in the $0.5-4 \mathrm{THz}$ range, popular inorganic nonlinear crystals, such as GaP, ZnTe and $\mathrm{LiNbO}_{3}$, have maximum values of $<28 \mathrm{pm} \mathrm{V}^{-1}$ and other organic crystals, such as DAST, DSTMS and $\mathrm{OH} 1$, have values of $<52 \mathrm{pm} \mathrm{V}^{-1}$. Furthermore, large $\left(5 \times 5 \mathrm{~mm}^{2}\right.$ cross-section) crystals of DPFO can be grown and exhibit a large laser damage threshold. Terahertz generation and detection experiments based on optical rectification with DPFO crystals indicate that these crystals work well for these purposes with a strong narrow-band response. $O G$

https://doi.org/10.1038/s41566-018-0125-4

\section{PT SYMMETRY}

\section{Exceptionally slow light}

Phys. Rev. Lett. 120, 013901 (2018)

The concept of stopping light in its tracks is one that has long captured the imagination of scientists. Now, Tamar Goldzak and co-workers from Brazil and Israel have theoretically demonstrated that the group velocity of light can tend to zero in parity-time (PT)symmetric systems. They show that zero group velocity may be achieved if a waveguide system is prepared at an exceptional point - a point where two optical modes coalesce with degenerate eigenfrequencies and eigenvectors. In their theory, they set up a system of a small waveguide array, coupling between a waveguide with gain and another with loss, to achieve a PT-symmetric system. The waveguide widths and distance between the waveguides are $\sim 1 \mu \mathrm{m}$. In principle, the effect and structural parameters can be adjusted for optical pulses in a wide range of frequencies and bandwidths. The approach can potentially be extended to acoustic and other types of wave.

https://doi.org/10.1038/s41566-018-0128-1 